\title{
Insectos escama (Hemiptera: Coccomorpha) en raíces de café de Norte de Santander y Valle del Cauca, Colombia y descripción de una nueva especie
}

\author{
Scale insects (Hemiptera: Coccomorpha) in coffee roots of Norte de Santander and Valle del Cauca, Colombia, \\ and description of a new species \\ ALEJANDRO CABALLERO ${ }^{1}$, ANDREA AMALIA RAMOS-PORTILLA², \\ ZULMA NANCY GIL ${ }^{3}$ y PABLO BENAVIDES ${ }^{4}$
}

\begin{abstract}
Resumen: Actualmente los insectos escama hipogeos (Hemiptera: Coccomorpha) son el segundo artrópodo plaga más importante de la caficultura colombiana. Para desarrollar planes de manejo integrado de este problema fitosanitario se hace necesario reconocer las especies que están asociadas al cultivo. En esta investigación se analizaron 768 especímenes de 85 muestras recolectadas en raíces de café en Norte de Santander y Valle del Cauca. Se identificaron 10 géneros reunidos en cuatro familias. Puto barberi, Toumeyella coffeae y Dysmicoccus varius fueron las especies más recurrentes. Se registra a Dysmicoccus mackenziei, Dysmicoccus radicis y Mixorthezia minima por primera vez para Colombia y a Coffea arabica como nuevo hospedante de Phenacoccus solani y se describe una nueva especie del género Pseudorhizoecus.
\end{abstract}

Palabras clave: Insectos plaga, Coccoidea, Orthezioidea.

\begin{abstract}
Currently, the hypogeal scale insects (Hemiptera: Coccomorpha) are the second most important problem pest arthropods for Colombian coffee crops. In order to develop integrated pest management programs, it is necessary to identify the species associated with this crop. In this study, 768 specimens from 85 samples collected from coffee roots in Norte de Santander and Valle del Cauca were analyzed. Ten genera belonging to four families were identified. Puto barberi, Toumeyella coffeae and Dysmicoccus varius were the most recurrent species. Dysmicoccus mackenziei, Dysmicoccus radicis and Mixorthezia minima are recorded for first time in Colombia. Coffea arabica is reported to be a new host for Phenacoccus solani and a new species of the genus Pseudorhizoecus is described.
\end{abstract}

Key words: Insects pests, Coccoidea, Orthezioidea.

\section{Introducción}

Los insectos escama (Hemiptera: Coccomorpha) son interesantes por su impacto en las actividades agrícolas (Dhaliwal et al. 2010): excretan sustancias azucaradas que sirven de medio de crecimiento de hongos causantes de fumagina, se alimentan de savia elaborada, poseen protección cerosa que los protege del ambiente y de los insecticidas, tienen asociación con hormigas, permiten el ingreso de patógenos y, en algunos casos, trasmiten toxinas o virus (McKenzie 1967; Kosztarab y Kozár 1988; Williams y Granara de Willink 1992; Gullan 1997; Gullan y Kosztarab 1997; Ramos y Serna 2004; Kondo et al. 2008a).

A nivel mundial, los insectos escama constituyen un importante grupo de plagas del café (Coffea spp.) (James 1935; Le Pelley 1973; Watson y Cox 1990; Culik et al. 2006; Barrera 2008; Sousa et al. 2012). En Colombia, desde 1930 se consideran el segundo grupo más importante de insectos plaga en este cultivo (Federación Nacional de Cafeteros 1932), destacándose Puto antioquensis (Murillo, 1931) (Putoidae) y Planococcus citri (Risso, 1813) (Pseudococcidae). En la década de los 70, se encontró en altas poblaciones en cafetales de Antioquia y Caldas (Benavides-Gómez y Cárdenas-Murillo 1977) a Neochavesia caldasiae (Balachowsky, 1957) (Rhizoecidae). Recientemente, las poblaciones de cocomorfos de raíz se han incrementado, tanto en etapa de almácigo como en establecimiento del cultivo, generando, en algunos casos, la muerte de las plantas antes del inicio de su producción, lo que obliga a llevar a cabo resiembras que aumentan los costos de producción (Villegas et al. 2009; Villegas-García y Benavides-Machado 2011; Federación Nacional de Cafeteros 2013; Gil et al. 2015).

Las siguientes especies están registradas en raíces de café en Colombia: Toumeyella coffeae Kondo, 2013 de la familia Coccidae; Puto barberi (Cockerell, 1895) y P. antioquensis (Murillo, 1931) de Putoidae; Neochavesia caldasiae (Balachowsky, 1957), N. eversi (Beardsley, 1970), Rhizoecus americanus (Hambleton, 1946), R. coffeae Laing, 1925 y Geococcus coffeae Green, 1933 de Rhizoecidae y Dysmicoccus brevipes (Cockerell, 1893), D. caribensis Granara de Willink, 2009, D. neobrevipes Bearsdley, 1959, D. texensis (Tinsley, 1900), D. varius Granara de Willink, 2009, Planococcus citri (Risso, 1813) y Pseudococcus jackbeardsleyi Gimpel y Miller, 1996 de Pseudococcidae (Murillo 1931; Hambleton 1946; Balachowsky 1959; Benavides-Gómez y CárdenasMurillo 1977; Williams y Granara de Willink 1992; Gimpel y Miller 1996; Kondo 2001; Kondo et al. 2008b; Granara de Willink 2009; Villegas et al. 2009; Kondo 2013).

Pese a la importancia de estos insectos en el café y al desconocimiento taxonómico de las especies asociadas, hasta

\footnotetext{
${ }^{1}$ Ingeniero agrónomo. Facultad de Ciencias Agrarias, Universidad Nacional de Colombia. Museo Entomológico, UNAB, Bogotá, D. C., Colombia, lacaballeror@ unal.edu.co, alejandrocab66@gmail.com. ${ }^{2}$ Ph. D. Instituto Colombiano Agropecuario (ICA), Dirección Técnica de Sanidad Vegetal, Bogotá, D. C., Colombia, andrea.ramos@ica.gov.co, andreaamaliaramos@gmail.com. ${ }^{3} \mathrm{Ph}$. D. Disciplina de Entomología, Centro Nacional de Investigación de Café-Cenicafé, Chinchiná, Caldas, Colombia, zulma.gil@cafedecolombia.com. ${ }^{4} \mathrm{Ph}$. D. Disciplina de Entomología, Centro Nacional de Investigación de Café-Cenicafé, Chinchiná, Caldas, Colombia, pablo.benavides@cafedecolombia.com. Autor para correspondencia: Alejandro Caballero. Ingeniero agrónomo. Facultad de Ciencias Agrarias, Universidad Nacional de Colombia. Museo Entomológico UNAB, Bogotá, D. C., Colombia, lacaballeror@unal.edu.co,alejandrocab66@gmail.com.
} 
la fecha no se ha realizado un muestreo con los métodos adecuados que permita reconocer las especies que se alimentan de este cultivo. Por esta razón, el objetivo de esta investigación es aportar al conocimiento fitosanitario del cultivo de café en Colombia por medio de la determinación taxonómica de insectos escamas recolectados de raíces de café en los departamentos de Norte de Santander y Valle del Cauca.

\section{Materiales y métodos}

El muestreo se desarrolló por parte del Servicio de Extensión Rural de la Federación Nacional de Cafeteros (FNC), entre junio y septiembre de 2013. Los especímenes se recolectaron manualmente de raíces de plantas de café menores a 24 meses de establecimiento, en 73 fincas de los departamentos de Norte de Santander y Valle del Cauca. Los predios se seleccionaron aleatoriamente a partir del Sistema de Información Cafetera SICA de FNC. Las muestras se conservaron en etanol $(76 \%)$ y se rotularon con datos de campo.

El montaje de los especímenes en láminas para microscopía se realizó empleando el protocolo del Systematic Entomology Laboratory (SEL-USDA) (2014): evisceración con hidróxido de potasio al $10 \%$, deshidratación progresiva con etanol al 75, 86 y 96\%, tinción con fucsina ácida, aclarado con aceite de clavo y sellado permanente con bálsamo de Canadá. El estudio de morfología e identificación se realizó en el Museo Entomológico Agronomía Bogotá (UNAB) de la Facultad de Ciencias Agrarias Universidad Nacional de Colombia, en el Museo Entomológico "Marcial Benavides" (MEMB, Centro Nacional de Investigaciones de Café-Cenicafé), Landscape and Ornamental Pests Laboratory (Purdue University, EE.UU.) y SEL-USDA (EE.UU.). Para la observación macroscópica se emplearon estereomicroscopios Nikon MSZ-1. La observación microscópica se hizo en microscopios de contraste de fases Zeiss LSM 880, Olympus IX51 y Nikon Eclipse E6000. Las imágenes se tomaron y analizaron con los programas Axion Vision e Image-Pro Insigth 8.0.

Las identificaciones fueron corroboradas según su especialidad, por Dr. Takumasa Kondo (Corporación Colombiana para la Investigación-Corpoica, Colombia), Dra. Lucía Claps (Universidad de Tucumán, Argentina), Dr. Douglass Miller (USD-SEL, EE.UU.) y Scott Schneider (Universidad de Massachusetts, EE.UU.). Los especímenes estudiados y el material tipo de la especie descrita están depositados en el museo UNAB (números de catálogo para cada especie en la Tabla 1) y se dispusieron láminas de respaldo en MEMB.

La terminología morfológica sigue las propuestas de Hambleton (1946, 1977a, 1977b), Williams y Granara de Willink (1992), Kozár y Konczné (2007), Schneider y Lapolla (2011) y Ramos-Portilla y Caballero (2016). La descripción de la nueva especie se basa en la morfología externa de la hembra adulta. El material tipo está conformado por seis especímenes. Las medidas se presentan en orden holotipo y entre paréntesis paratipos; no todas las medidas consideran los seis especímenes. La longitud de antena, escudo clipeolabral, labio y trocánter corresponde a la parte media de cada segmento; la longitud de cada segmento de la pata corresponde a su margen externo y la longitud total de cada pata corresponde a la suma de fémur, tibia, tarso y uña. El largo y ancho de cada estructura corresponde a la sección más amplia marcada por una línea recta de cada segmento.

\section{Resultados y discusión}

Se procesaron e identificaron 768 especímenes a partir de 85 muestras. Se determinaron 21 especies en 12 géneros y cinco familias (Tabla 1). Pseudococcidae fue la familia más representativa con 10 especies, seguida por Rhizoecidae (7), Coccidae (1), Ortheziidae (1) y Putoidae (1). Estos resultados son los esperados, dado que las especies de Pseudococcidae tienen una alta plasticidad biológica que les permite habitar en estructuras aéreas y subterráneas y alimentarse de diferentes tejidos de la planta (McKenzie 1967; Williams y Granara de Willink 1992), de allí que existan cerca de 314 especies asociadas a raíces (McKenzie 1967; Williams 1985; Watson y Cox 1990; Ben-Dov 1994; Gimpel y Miller 1996; Kosztarab 1996; Miller y Arapu 1997; Hara et al. 2001; Culik et al. 2007; Danzig 2007; Kondo et al. 2008b; Granara de Willink 2009; Kaydan y Gullan 2012; Malumphy 2014; García-Morales et al. 2016; Gavrilov-Zimin y Kurochkin 2016), mientras que Rhizoecidae está compuesta exclusivamente por especies rizófagas (Hambleton 1946; Kozár y Konczné 2007; Schneider y Lapolla 2011).

Por otro lado, aunque Coccidae es la tercera familia más diversa de Coccomorpha (Ben-Dov y Hodgson 1997; GarcíaMorales et al. 2016), las especies que la componen tienden a alimentarse de estructuras aéreas de la planta (Kosztarab y Kozár 1988), muestra de ello es que solo 32 especies han sido registradas alimentándose de tejido radical (Gill 1988; Kosztarab y Kozár 1988; Ben-Dov 1994; Kosztarab 1996; Kondo y Gullan 2004; Ben-Dov 2012; Kondo 2013; Moghaddam 2013; García-Morales et al. 2016). El número bajo de especies de Ortheziidae y Putoidae probablemente se debe a que son familias compuestas por menos especies respecto a Pseudococcidae y Coccidae y todavía existen vacíos en la información de su ecología y biología (Kozár 2004; Williams et al. 2011; Vea 2014). Por otra parte, Rhizoecidae tiene como mayor centro de diversidad el Neotrópico, mientras que para Ortheziidae es el Paleartico (Kozár 2004; Kozár y Konczné 2007).

Respecto a los hallazgos a nivel de género, en las cochinillas harinosas (Pseudococcidae, Putoidae y Rhizoecidae) se observa que los géneros con más especies correspoden a aquellos que mundialmente son más diversos, como Dysmicoccus Ferris, Puto Signoret y Rhizoecus Künckel d'Herculais (Hambleton 1976; Williams y Granara de Willink 1992; Kozár y Konczné 2007; Granara de Willink 2009; Williams et al. 2011; Ramos-Portilla y Caballero 2016).

Los resultados a nivel de especie muestran a Puto barberi como la más recurrente, presente en 64 (75\%) de las 85 muestras; este porcentaje es similar a los resultados reportados por Villegas-García y Benavides-Machado (2011) para los departamentos de Caldas, Cauca, Cundinamarca, Norte de Santander, Risaralda, Santander y Tolima, lo cual hace pensar que $P$. barberi es la especie con mayor distribución en Colombia. Toumeyella coffeae y Dysmicoccus varius representan el $40 \%$ del total de los especímenes analizados. Estas especies no están registradas en trabajos previos, excepto aquellos en donde se describen, por lo que su hallazgo muestra que son potenciales nuevas plagas del café.

Se hace el primer registro de Dysmicoccus mackenziei, $D$. radicis y Mixorthezia minima para Colombia. Dysmicoccus mackenziei se lista con amplia distribución en Centro América y en Ecuador mientras que $D$. radicis tiene distribución restringida en Sur América (Surinam y Brasil) (Williams y 
Granara de Willink 1992; Granara de Willink 2009) y M. minima está registrada para Brasil (Kozár 2004).

Se registra por primera vez a M. minima y Phenacoccus solani Ferris asociadas a café. En el caso de M. minima no se registra información de asociación con plantas, aunque Kozár (2004) menciona haber recolectado los especímenes en musgo y hojarasca. Ph. solani está asociada a 29 familias botánicas, pero no existen registros de especies de Rubiaceae

Tabla 1. Listado de especies de insectos escama asociados a raíces de café en Norte de Santander (NS) y Valle del Cauca (VC). Se referencia información de la familia, especie, departamento donde fue recolectado en este estudio, además del número de catálogo del Museo Entomológico UNAB, distribución y hospedantes.

\begin{tabular}{|c|c|c|c|c|c|}
\hline Taxón & Departamento & $\begin{array}{l}\text { No Cat. } \\
\text { UNAB }\end{array}$ & Distribución & Hospedante & $\begin{array}{c}\text { Referencia } \\
\text { bibliográfica }\end{array}$ \\
\hline \multicolumn{6}{|l|}{ Coccidae } \\
\hline Toumeyella coffeae Kondo, 2013 (Fig. 1A) & $\mathrm{NS}, \mathrm{VC}$ & 1412 & Neotrópico & Coffea sp. & 32,36 \\
\hline \multicolumn{6}{|l|}{ Ortheziidae } \\
\hline $\begin{array}{l}\text { Mixorthezia minima Konczné y Kozár, } 2004^{1,2} \\
\text { (Fig. 1B) }\end{array}$ & NS & 1806 & $\begin{array}{l}\text { Neotrópico } \\
\left(\text { Colombia }{ }^{1}\right)\end{array}$ & $\begin{array}{c}\text { Suelo, } \\
\text { Coffea arabica }\end{array}$ & 22 \\
\hline \multicolumn{6}{|l|}{ Pseudococcidae } \\
\hline Dysmicoccus brevipes (Cockerell, 1893) & $\mathrm{NS}, \mathrm{VC}$ & 1390 & Cosmopolita & [Polífaga] & $\begin{array}{c}5,6,13,14,15,23,26 \\
30,31,34,37\end{array}$ \\
\hline Dysmicoccus caribensis Granara de Willink, 2009 & $\mathrm{NS}, \mathrm{VC}$ & 1391 & Neotrópico & Café & 31 \\
\hline $\begin{array}{l}\text { Dysmicoccus mackenziei Beardsley, } 1965^{1} \\
\text { (Fig. 1C) }\end{array}$ & NS & 1393 & $\begin{array}{l}\text { Cosmopolita } \\
\left(\text { Colombia }^{1}\right)\end{array}$ & [Polífaga $]$ & $6,8,14,15,31$ \\
\hline Dysmicoccus neobrevipes Beardsley, 1959 & $\mathrm{VC}$ & 1394 & Cosmopolita & [Polífaga $]$ & $\begin{array}{c}10,14,15,19,20,30 \\
31,35\end{array}$ \\
\hline $\begin{array}{l}\text { Dysmicoccus radicis (Green, 1933) }{ }^{1} \\
\text { (Fig. 1D) }\end{array}$ & NS & 1399 & $\begin{array}{l}\text { Neotrópico } \\
\left(\text { Colombia }^{1}\right)\end{array}$ & $\begin{array}{c}\text { Coffea sp., Cyperus } \\
\text { hermaphroditus } \\
\text { (Jacq.) Standl., } 1916\end{array}$ & $1,15,31$ \\
\hline Dysmicoccus texensis (Tinsley, 1900) & $\mathrm{VC}$ & 1397 & $\begin{array}{l}\text { Neártico, } \\
\text { Neotrópico }\end{array}$ & [Polífaga] & $6,14,15,19,21,30,31$ \\
\hline $\begin{array}{l}\text { Dysmicoccus varius Granara de Willink, } 2009 \\
\text { (Fig. 1E) }\end{array}$ & NS & 1395 & Neotrópico & Coffea sp. & 31 \\
\hline $\begin{array}{l}\text { Phenacoccus solani Ferris, } 1918^{2} \\
\text { (Fig. 1F) }\end{array}$ & $\mathrm{VC}$ & 1404 & Cosmopolita & $\begin{array}{c}\text { [Polífaga }], \\
\text { Coffea arabica }\end{array}$ & $6,14,15,17,30,37$ \\
\hline Planococcus minor (Maskell, 1897) & $\mathrm{VC}$ & 1403 & Cosmopolita & [Polífaga] & $\begin{array}{c}12,14,15,21,30,37 \\
38\end{array}$ \\
\hline Pseudococcus elisae Borchsenius, 1947 & $\mathrm{NS}, \mathrm{VC}$ & 1405 & Neotrópico & [Polífaga] & $11,14,16,27$ \\
\hline Pseudococcus landoi (Balachowsky, 1959) & $\mathrm{VC}$ & 1406 & $\begin{array}{l}\text { Neártico, } \\
\text { Neotrópico }\end{array}$ & [Polífaga] & $4,14,15,16$ \\
\hline \multicolumn{6}{|l|}{ Putoidae } \\
\hline Puto barberi (Cockerell, 1895) (Fig. 1G) & $\mathrm{NS}, \mathrm{VC}$ & 1408 & Neotrópico & [Polífaga] & $6,14,15,19,30,33$ \\
\hline \multicolumn{6}{|l|}{ Rhizoecidae } \\
\hline Geococcus coffeae Green, 1933 (Fig. 1H) & $\mathrm{NS}, \mathrm{VC}$ & 1400 & Cosmopolita & [Polífaga] & $1,7,25,28,29$ \\
\hline $\begin{array}{l}\text { Neochavesia caldasiae (Balachowsky, 1957) } \\
\text { (Fig. 1I) }\end{array}$ & $\mathrm{VC}$ & 1401 & Neotrópico & $\begin{array}{c}\text { Musa sp., } \\
\text { Coffea arabica }\end{array}$ & $3,18,24,29,32$ \\
\hline $\begin{array}{l}\text { Pseudorhizoecus bari n. sp. Caballero y Ramos } \\
\text { (Fig. 1J) }\end{array}$ & NS & 1807 & Colombia & Coffea arabica & \\
\hline Rhizoecus americanus (Hambleton, 1946) & $\mathrm{VC}$ & 1326 & Cosmopolita & [Polífaga] & $\begin{array}{c}2,9,14,15,19,25 \\
29,39\end{array}$ \\
\hline Rhizoecus coffeae Laing, 1925 & NS & 1409 & Neotrópico & [Polífaga] & $1,14,15,29$ \\
\hline $\begin{array}{l}\text { Rhizoecus colombiensis Ramos y Caballero, } 2016 \\
\text { (Fig. } 1 \mathrm{~K} \text { ) }\end{array}$ & $\mathrm{VC}$ & 678 & $\begin{array}{l}\text { Neártico, } \\
\text { Neotrópico }\end{array}$ & [Polífaga] & 40 \\
\hline Ripersiella andensis (Hambleton, 1946) & $\mathrm{VC}$ & 1411 & Neotrópico & $\begin{array}{l}\text { Musa sp., } \\
\text { Coffea arabica }\end{array}$ & $2,14,15,29$ \\
\hline
\end{tabular}

${ }^{1}$ Nuevo registro de especie para el país; ${ }^{2}$ Nuevo registro de hospedante. Referencias bibliográficas: 1. Green (1933). 2. Hambleton (1946). 3. Balachowsky (1957). 4. Balachowsky (1959). 5. Beardsley (1959). 6. McKenzie (1967). 7. Williams (1968). 8. Williams y McKenzie (1971). 9. Hambleton (1976). 10. Beardsley et al. (1982). 11. Beardsley (1986). 12. Cox (1989). 13. Orellana y Vera (1989). 14. Williams y Granara de Willink (1992). 15. Ben-Dov (1994). 16. Gimpel y Miller (1996). 17. Kosztarab (1996). 18. Johnson et al. (2001). (19. Kondo (2001). (20. Miller y Williams (2002). 21. Santa-Cecilia et al. (2002). 22. Kozár (2004). 23. Ramos y Serna (2004). 24. Williams (2004a). 25. Williams (2004b). 26. Watson y Kubiriba (2005). 27. Culik et al. (2006). 28. Kianek et al. (2007). 29. Kozár y Konczné (2007). 30. Kondo et al. (2008b). 31. Granara de Willink (2009). 32. Villegas-García y Benavides-Machado (2011). 33. Williams et al. (2011). 34. Ben-Dov (2012). 35. Tanaka y Uesato (2012). 36. Kondo (2013). 37. Moghaddam (2013). 38. Malumphy (2014). 39. Ramos-Portilla et al. (2014). 40. Ramos-Portilla y Caballero (2016). 
como hospedante (Williams y Granara de Willink 1992; BenDov 1994; Granara de Willink y Szumik 2007).

\section{Rhizoecidae Hodgson, 2012}

\section{Pseudorhizoecus bari n. sp. Caballero y Ramos (Fig. 1J)}

Material tipo. Pseudorhizoecus bari n. sp. HOLOTIPO: $ᄋ$ adulta, en raíces de café, Coffea arabica L., 1753 (Rubiaceae). COLOMBIA. Norte de Santander. Convención, Vda. El Retiro, Fca. Monte Cristo, $1242 \mathrm{msnm}$. Captura manual. 11-jun-2013 [UNAB]. PARATIPOS: 3 adultas $+2 q$ de tercer instar ninfal, datos iguales al holotipo [UNAB]. Machos no observados.
Etimología. Esta especie es nombrada en homenaje a la etnia indígena Barí, quienes habitan la región donde se recolectó el material tipo, nombre en aposición.

Descripción. En vivo de color blanco o blanco cremoso, circular, sin recubrimiento de cera (Fig. 1J). En lámina de microscopia: Cuerpo (Fig. 2) subcircular, 1,2 (0,7-1,1) mm de largo, $1(0,5-0,9) \mathrm{mm}$ de ancho. Órgano genital visible en dos paratipos (75 y $87 \mu \mathrm{m}$ de largo).

Dorso. Ostiolos ausentes. Ano: anillo anal circular u ovalado, 80 (67-75) $\mu \mathrm{m}$ de diámetro, rodeado por un área esclerosada delgada con 23 (21) setas flageladas, 35-60 $\mu \mathrm{m}$ de largo, sin celdas, con dos o tres protuberancias. Lóbulos anales
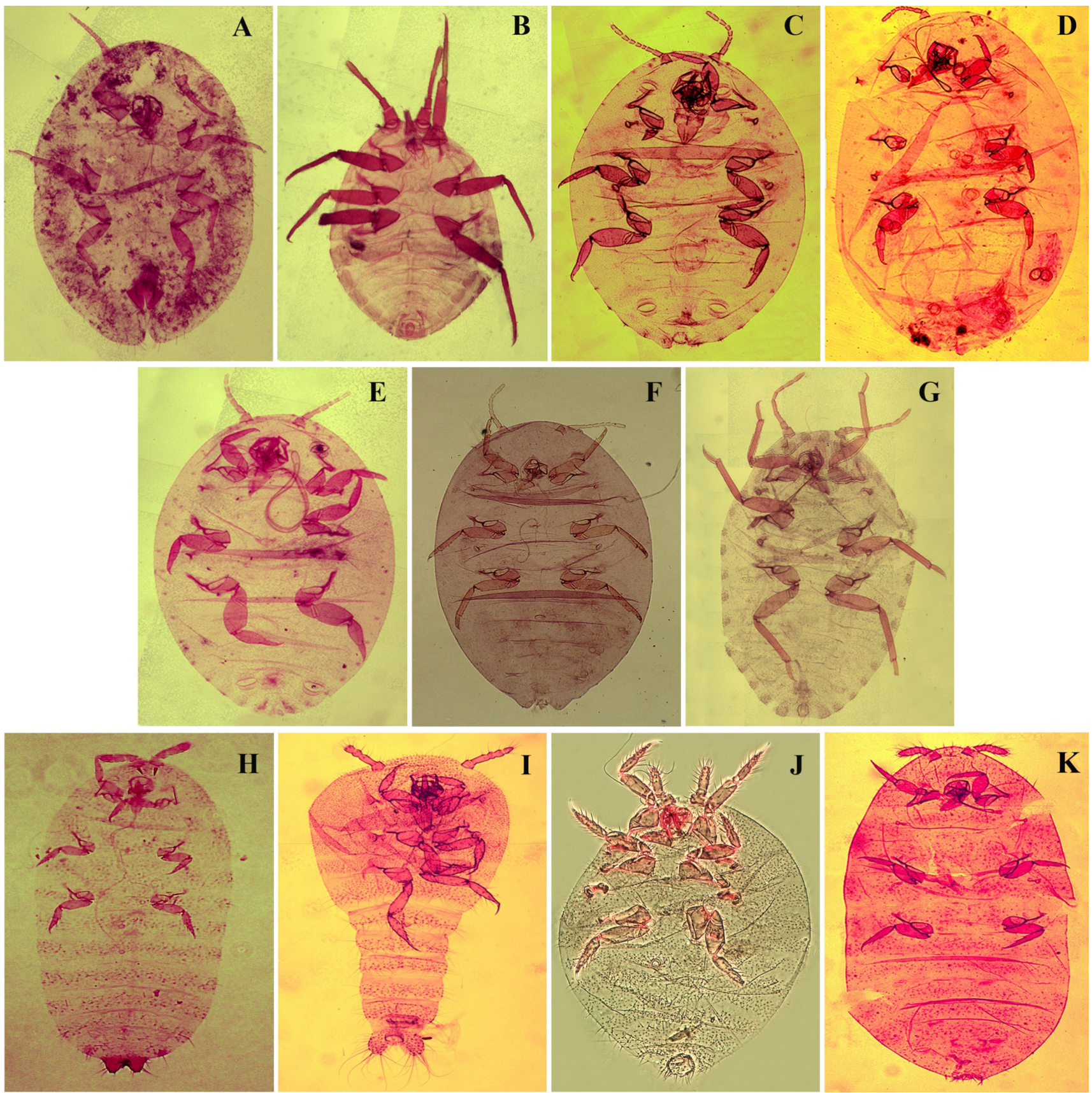

Figura 1. Hábitus de A. Toumeyella coffeae. B. Mixorthezia minima. C. Dysmicoccus mackenziei. D. Dysmicoccus radicis. E. Dysmicoccus varius. F. Phenacoccus solani. G. Puto barberi. H. Geococcus coffeae. I. Neochavesia caldasiae. J. Pseudorhizoecus bari n. sp. K. Rhizoecus colombiensis. 


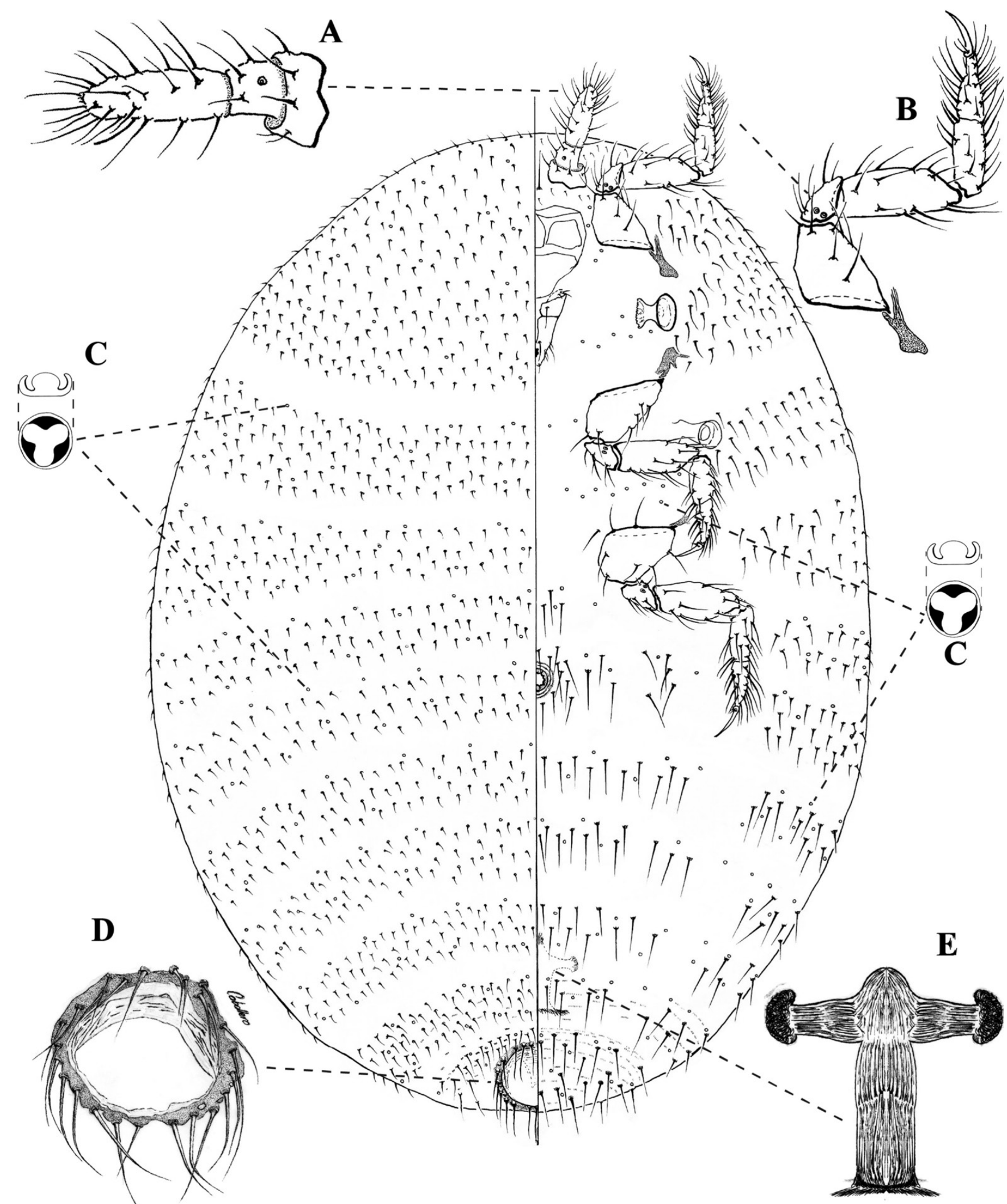

Figura 2. Pseudorhizoecus bari n. sp. Caballero y Ramos, hembra adulta. Ampliación de: A. Antena. B. Pata anterior. C. Poro trilocular. D. Anillo anal. E. Cámara genital.

no diferenciables en Holotipo y ligeramente desarrollados en hembras adultas tenerales, con setas no diferenciables a otras del segmento. Setas corporales flageladas, en bandas intrasegmentales desde mesotórax hasta cauda; cefálicas y protorácicas en un único grupo; longitud de setas en últimos segmentos abdominales progresivamente más largas. Conductos tubulares, bitubulares y tritubulares ausentes. Poros triloculares 3-4 $\mu \mathrm{m}$ de diámetro, distribuidos en toda la su- 
perficie; poros discoidales y multiloculares ausentes. Espículas presentes, evidentes en abdomen posterior.

Vientre. Ojos ausentes. Placa cefálica no visible. Antena de tres segmentos, 127 (130-133) $\mu \mathrm{m}$ de largo: segmento I: largo 33 (29-32) $\mu \mathrm{m}$, ancho 53 (47-53) $\mu \mathrm{m}$, cinco setas flageladas; segmento II: largo 21 (20-21) $\mu \mathrm{m}$, ancho 31 (3135) $\mu \mathrm{m}$, tres setas flageladas y un sensorio campaniforme; segmento III: largo 82 (80-81) $\mu \mathrm{m}$, ancho 27 (28-31) $\mu \mathrm{m}$, dos setas de ápice truncado, apicales, $5 \mu \mathrm{m}$ de largo, dos sensorios filiformes delgados, de ápice redondeado, distales, en margen externo, $33 \mu \mathrm{m}$ de largo, 23 setas flageladas. Escudo clipeolabral: largo $102 \mu \mathrm{m}$, ancho $108 \mu \mathrm{m}$. Labio de tres segmentos: basal membranoso, medio y apical esclerosados; tres, uno y siete pares de setas flageladas respectivamente; largo $96 \mu \mathrm{m}$. Patas caminadoras; coxas con 11 setas flageladas; trocánteres con cinco setas flageladas y cuatro sensorios campaniformes; todos los tarsos con una sensorio campaniforme basal en margen externo; uñas con digítulos setosos, delgados, más cortos que la uña, 10-12 $\mu \mathrm{m}$ de largo, dentículo ausente. Propatas: largo 273 (233289) $\mu \mathrm{m}$; fémur: largo 106 (109-115) $\mu \mathrm{m}$, ancho 42 (38-44) $\mu \mathrm{m}, 15$ setas flageladas; tibia: largo $53(65-73) \mu \mathrm{m}$, ancho $30(26-30) \mu \mathrm{m}, 14$ setas flageladas, en filas longitudinales y dos espuelas preapicales en margen interno; tarso: largo 49 (49-51) $\mu \mathrm{m}$, ancho 25 (22-25) $\mu \mathrm{m}, 20$ setas flageladas, en filas longitudinales y una espuela en margen interno; uña: largo 52 (48-50) $\mu \mathrm{m}$. Mesopata: largo 281 (274-286) $\mu \mathrm{m}$ : fémur: largo 111 (108-115) $\mu \mathrm{m}$, ancho 41 (44-50) $\mu \mathrm{m}, 15$ setas flageladas; tibia: largo $68(62-70) \mu \mathrm{m}$, ancho $32(28$ 30) $\mu \mathrm{m}, 15$ setas flageladas y dos espuelas preapicales marginales internas (una más robusta que la otra); tarso: largo 52 (50-52) $\mu \mathrm{m}$, ancho 25 (23-25) $\mu \mathrm{m}, 20$ setas flageladas, una espuela preapical en margen interno; uña: largo 50 (4752) $\mu \mathrm{m}$. Metapata: largo 287 (287-297) $\mu \mathrm{m}$; fémur: largo 106 (114-117) $\mu \mathrm{m}$, ancho 48 (45-54) $\mu \mathrm{m}, 16$ setas flageladas; tibia: largo 67 (66-74) $\mu \mathrm{m}$, ancho 33 (32-38) $\mu \mathrm{m}, 16$ setas flageladas y dos espuelas preapicales en margen interno; tarso: largo 56 (52-56) $\mu \mathrm{m}$, ancho 25 (24-28) $\mu \mathrm{m}$, cada una con 19 setas flageladas y una espuela proximal en margen interno; uña: largo 58 (50-55) $\mu \mathrm{m}$. Círculo en segmento abdominal III, protruido, en forma de cono truncado, cima irregular, diámetro basal 42 (40-42) $\mu \mathrm{m}$, diámetro apical 30 (21-28) $\mu \mathrm{m}$. Vulva mesial entre segmentos abdominales VII y VIII (evidente en paratipos).

Poros triloculares: diámetro 3-4 $\mu \mathrm{m}$; distribuidos en la superficie. Poros multiloculares y discoidales ausentes. Conductos tubulares, bitubulares y tritubulares ausentes. Setas corporales flageladas: en cabeza, dos setas flageladas, anteromesiales, de 35 (35-42) $\mu \mathrm{m}$, en el espacio interantenal, las demás setas son flagelas, conspicuamente más cortas que las anteriormente descritas; en tórax, ausentes en área mesial, submesial y áreas intersegmentales, presentes en áreas submarginales y marginales, formando grupos en cada segmento torácico, el grupo del segmento protorácico se une a las setas cefálicas; en abdomen se presentan dos tipos de setas flageladas: I) setas conspicuamente largas $(30-40 \mu \mathrm{m})$, rectas y robustas presentes en mesón y submesón, en filas transversales a cada segmento, II) setas cortas $(5-10 \mu \mathrm{m})$, curvas y delgadas, intrasegmentales desde el submargen hasta el margen, ausentes de áreas laterales y espacios intersegmentales. Espículas presentes en toda la superficie.
Diagnosis. Pseudorhizoecus bari n. sp. puede ser diferenciado de Pseudorhizoecus proximus Green, 1933 porque el primero presenta antena con tres segmentos, círculo presente y anillo anal con más de 20 setas flageladas; en tanto que $P$. proximus presenta antenas de 5 segmentos, círculo ausente y anillo anal con 2-5 setas. También puede ser confundido con Ripersiella colombiensis (Hambleton, 1946) por la forma del cuerpo, la abundancia de setas corporales y la presencia de círculo; sin embargo, R. colombiensis posee antenas con 5 segmentos y un anillo anal con seis setas flagelas y dos bandas de celdas anales evidentes.

Esta especie presenta tres atributos poco frecuentes dentro de la familia Rhizoecidae: ausencia de conductos de cualquier tipo, antena de tres segmentos y anillo anal sin celdas, con más de 20 setas rodeando la apertura. Dentro de la subfamilia Rhizoecinae Williams, donde se ubica Pseudorhizoecus Green, la ausencia de conductos tubulares, bi o tritubulares es característico únicamente de géneros monotípicos como Brevicoccus Hambleton, Capitisetella Hambleton y Lepthorhizoecus Williams y la especie $R$. colombiensis (Green 1933; Hambleton 1946; Hambleton 1977a; Williams 1998; Kozár y Konczné 2007), es decir, solo en cinco especies de las cerca de 200 que componen a la subfamilia. Por otro lado, en la misma subfamilia no existen especies descritas con antenas de tres segmentos; este atributo se presenta sólo en el género Eumyrmococcus Silvestri, perteneciente a la subfamilia Xenococcinae (Tang) (Kozár y Konczné 2007; Schneider y Lapolla 2011). El anillo carente de celdas también es atípico, presente solo en $P$. proximus pero este último posee 2-5 setas anales (Green 1933; Williams 1998; Kozár y Konczné 2007; Schneider y Lapolla 2011).

Pseudorhizoecus Green, antes de esta publicación, pertenecía a un grupo de géneros monotípicos de la subfamilia Rhizoecinae; su especie Tipo, $P$. proximus Green, está restringida a la región Neotropical y está registrada en Colombia, Costa Rica, Ecuador, Guatemala y Surinam; asociada con Coffea sp., C. arabica, C. liberica, Musa sapientum, Musa sp. y Theobroma cacao (Williams y Granara de Willink 1992; Kozár y Konczné 2007).

Debido a los argumentos arriba explicados, la clasificación taxonómica de Pseudorhizoecus bari n. sp. está abierta a la discusión; su ubicación temporal dentro de Pseudorhizoecus se debe a la semejanza en la mayoría de caracteres morfológicos, datos de ubicación y hospedantes. Se espera que el incremento de especímenes recolectados, a través de muestreos más detallados, puedan ayudar a la clarificación de la ubicación taxonómica de esta especie.

\section{Conclusiones}

Esta investigación proporciona información necesaria para actualizar la lista de especies asociadas a la rizósfera de café. Actualmente Puto barberi es la especie de cocomorfos, de hábito rizófago, de mayor distribución en la caficultura de los departamentos de Norte de Santander y Valle del Cauca.

Especies de reciente descripción como Dysmicoccus varius y Toumeyella coffeae, están presentando una distribución más amplia, en comparación con investigaciones recientes donde $D$. texensis y $D$. brevipes eran las más distribuidas; esto demuestra que las dinámicas poblacionales de cocomorfos son variables espacio-temporalmente. El hábito hipogeo de estos insectos los hace pasar desapercibidos y son difíciles 
de detectar en campo; sin embargo, los nuevos registros para el cultivo del café y para Colombia, soportan la idea de Kozár y Konczné (2007) y Villegas et al. (2009) que los insectos escama de raíces son insectos que aún carecen de estudios de taxonomía. Esta investigación puede ser un ejemplo metodológico para conocer la fauna de cocomorfos hipogeos asociados a cultivos.

\section{Agradecimientos}

Agradecimientos especiales al Servicio de Extensión Rural de la Federación Nacional de Cafeteros de Colombia de los departamentos del Valle del Cauca y Norte de Santander por su invaluable colaboración en la recolección de las muestras. Al Museo Entomológico UNAB y su director, el Dr. Francisco Serna y a la Facultad de Ciencias Agrarias de la Universidad Nacional, sede Bogotá por el financiamiento y la infraestructura prestada. Al Dr. Takumasa Kondo, Dr. Scott Schneider y la Dra. Lucia Claps por la colaboración en la confirmación de identificaciones. El primer autor agradece al Dr. Greg Evans, Dr. Douglass Miller y a su esposa Barbara Denno (SEL-USDA); a Carlos Quesada, Dr. Clifford Sadof y Dr. Douglas Richmond (Purdue University) por su ayuda y hospitalidad durante la estancia de investigación en Estados Unidos. Los autores agradecen a los revisores anónimos por sus aportes al documento.

\section{Literatura citada}

BALACHOWSKY, A. S. 1957. Sur un nouveau genre aberrant de cochenille radicole myrmécophile nuisable au caféier en Colombie. Revue de Pathologie Végétale et d'Entomologie Agricole de France 36 (1): 157-164.

BALACHOWSKY, A. S. 1959. Nuevas cochinillas de Colombia I. Revista de la Academia Colombiana de Ciencias Exactas, Físicas y Naturales 10 (41): 337-361.

BARRERA, J. F. 2008. Coffee Pests and their Management. pp. 9619-9698. En: Capinera, J. L. (Ed.). Encyclopedia of Entomology. Springer, Tapachula. 4346 p.

BEARDSLEY, J. W. 1959. On the taxonomy of pineapple mealybugs in Hawaii, with a description of a previously unnamed species (Homoptera: Pseudococcidae). Proceedings of the Hawaiian Entomological Society 17 (1): 29-37.

BEARDSLEY, J. W. 1986. Taxonomic Notes on Pseudococcus elisae Borchsenius, a mealybug new to the Hawaiian fauna (Homoptera: Pseudococcidae). Proceedings of the Hawaiian Entomological Society 26 (1): 31-34.

BEARDSLEY, J. W.; SU, T. H.; MCEWEN, F. L.; GERLING, D. 1982. Field investigations on the interrelationship of the bigheaded ant, the gray pinneapple mealybug, and pinneapple mealybug wit disease in Hawaii. Proceedings of the Hawaiian Entomological Society 24 (1): 51-67.

BENAVIDES-GÓMEZ, M.; CÁRDENAS-MURILLO, R. 1977. Hormigas de Amagá y de la Esperanza. Avances Técnicos Cenicafé 69: 123-126.

BEN-DOV, Y. 1994. A systematic catalogue of the mealybugs of the world (Insecta, Homoptera, Coccoidea, Pseudococcidae and Putoidae): with data on geographical distribution, host plants, biology, and economic importance. Intercept Limited Andover, Reino Unido. $686 \mathrm{p}$

BEN-DOV, Y. 2012. The scale insects (Hemiptera: Coccoidea) of Israel-checklist, host plants, zoogeographical considerations and annotations on species. Israel Journal of Entomology 42 (1): 21-48.

BEN-DOV, Y.; HODGSON, C. J. 1997. Soft scale insects their biology, natural enemies and control. World Crop Pests Volume 7A. Elsevier Science, Amsterdam. 452 p.
COX, J. M. 1989. The mealybugs genus Planoccoccus (Homoptera: Pseudococcidae). Bulletin of the British Museum (Natural History) 58 (1): 1-78.

CULIK, M. P.; MARTINS, D. S.; GULLAN, P. J. 2006. First records of two mealybug species in Brazil and new potential pests of papaya and coffee. Journal of Insect Science 23 (1): 1-6.

CULIK, M. P.; MARTINS, D. S.; VENTURA, J. A.; PERONTI, A. L. B.; GULLAN, P. J; KONDO, T. 2007. Coccidae, Pseudococcidae, Ortheziidae, and Monophlebidae (Hemiptera: Coccoidea) of Espírito Santo, Brazil. Biota Neotropical 7 (3): 61-65.

DANZIG, E. M. 2007. Mealybugs of the Genus Fonscolombia Licht. (Homoptera, Pseudococcidae) of the fauna of Russia and adjacent countries. Entomological Review 87 (2): 547560.

DHALIWAL, G.; JINDAL, V.; DHAWAN, A. 2010. Insect pest problems and crop losses: changing trends. Indian Journal of Ecology 37 (1): 1-7.

FEDERACIÓN NACIONAL DE CAFETEROS. 1932. Manual del Cafetero Colombiano. Litografía Colombia, Bogotá D.C. 400 p.

FEDERACIÓN NACIONAL DE CAFETEROS. 2013. Manual del cafetero colombiano. Tomo II. Federación Nacional de Cafeteros, Chinchiná, Caldas. 354 p.

GARCÍA-MORALES, M.; DENNO, B.; MILLER, D.R.; MILLER, G. L.; BEN-DOV, Y.; HARDY, N. B. 2016. ScaleNet: A literature-based model of scale insect biology and systematics. Database. Disponible en: http://scalenet.info [Fecha revisión: 10 octubre 2016].

GAVRILOV-ZIMIN, I. A.; KUROCHKIN, A. S. 2016. Scale insects of the Volga area (Homoptera: Coccinea). IV. Mirococcus vallis sp. nov. and some other mealybugs (Pseudococcidae) from Samara Province of Russia. Zoosystemica Rossica 25 (1): 151156.

GIL, Z. N.; BENAVIDES, P.; VILLEGAS, C. 2015. Manejo integrado de las cochinillas de las raíces del café. Avances Técnicos $\mathrm{N}^{\circ} 459$. Cenicafé, $8 \mathrm{p}$.

GILL, R. J. 1988. The scale insects of California Part I: The soft scales (Homoptera: Coccoidea: Coccidae) 1a ed., California: California Department of Food and Agriculure. 142 p.

GIMPEL, W. F.; MILLER, D. R. 1996. Systematic analysis of the mealybugs in the Pseudococcus maritimus complex (Homoptera: Pseudococcidae). Contributions on Entomology, International 2 (1): 1-163.

GRANARA DE WILLINK, M. C. 2009. Dysmicoccus de la Región Neotropical (Hemiptera: Pseudococcidae). Revista de la Sociedad Entomológica Argentina 68 (1-2): 11-95.

GRANARA DE WILLINK, M. C.; SZUMIK, C. 2007. Phenacoccinae de Centro y Sudamérica (Hemiptera: Coccoidea: Pseudococcidae): Sistemática y Filogenia. Revista de la Sociedad Entomológica Argentina 66 (1-2): 29-129.

GREEN, E. E. 1933. Notes on some Coccidae from Surinam, Dutch Guiana, with descriptions of new species. Stylops 2 (3): 49-58.

GULLAN, P. J. 1997. 1.3.5 Relationships with ants. pp. 351-373. En: Ben-Dov, Y.; Hodgson, C. J. (Eds.). Soft scale insects Their biology, natural enemies and control. Elsevier Science, Amsterdam. 452 p.

GULLAN, P. J.; KOSZTARAB, M. 1997. Adaptations in Scale Insects. Annual Review of Entomology 42 (1): 23-50.

HAMBLETON, E. J. 1946. Studies of hypogeic mealybugs. Revista de Entomologia Rio de Janeiro 17 (1): 1-77.

HAMBLETON, E. J. 1976. A revision of the new world mealybugs of the genus Rhizoecus (Homoptera: Pseudococcidae). Technical Bulletin 1522: 1-88.

HAMBLETON, E. J. 1977a. A review of Pseudorhizoecus Green, with a description of a related new genus (Homoptera: Pseudococcidae). Journal of Washington Academy of Sciences 67 (1): 38-41.

HAMBLETON, E. J. 1977b. Notes on the species of Neorhizoecus Hambleton, a synonym of Rhizoecus Kunckel d'Herculais (Ho- 
moptera: Pseudococccidae). Proccedings of the Entomological Society of Washington 79 (1): 367-376.

HARA, A. H.; NIINO-DUPONTE, R. Y.; JACOBSEN, C. M. 2001. Root mealybugs of quarantine significance in Hawaii. College of Tropical Agriculture and Human Resources, IP-6: 1-4.

JAMES, H. C. 1935. New hypogeic mealybugs (Coccidae) from East Africa. Bulletin of Entomological Research 26 (3): 379390.

JOHNSON, C.; AGOSTI, D.; DELABIE, J. H.; DUMPERT, K.; WILLIAMS, D. J.; TSCHIRNHAUS, M. V; MASCHWITZ, U. 2001. Acropyga and Azteca ants (Hymenoptera: Formicidae) with scale insects (Sternorrhyncha: Coccoidea): 20 million years of intimate symbiosis. American Museum Novitates 3335 (1): 1-18.

KAYDAN, M. B.; GULLAN, P. J. 2012. A taxonomic revision of the mealybug genus Ferrisia Fullaway (Hemiptera: Pseudococcidae), with descriptions of eight new species and a new genus. Zootaxa 3543: 1-65.

KIANEK, A.; BENEDICTY, K.; KOZÁR, F.; BAYAR, K. H. 2007. A new species of Geococcus (Homoptera: Coccoidea: Pseudococcidae). Acta Zoologica Academiae Scientiarum Hungaricae 53 (1): 39-49

KONDO, T. 2001. Las Cochinillas de Colombia (Hemiptera: Coccoidea). Biota Colombiana 2 (1): 31-48.

KONDO, T. 2013. A new species of Toumeyella Cockerell (Hemiptera: Coccoidea: Coccidae) on coffee roots, Coffea arabica L. (Rubiaceae), from Colombia and Venezuela. Revista Corpoica Ciencia y Tecnología Agropecuaria 14 (1): 39-51.

KONDO, T.; GULLAN, P. J. 2004. A new species of ant-tended soft scale of the genus Cryptostigma Ferris (Hemiptera: Coccidae) associated with bamboo in Peru. Neotropical Entomology 33(6): 717-723.

KONDO, T.; GULLAN, P. J.; WILLIAMS, D. 2008a. Coccidology. The study of scale insects (Hemiptera: Sternorryncha: Coccoidea). Revista Corpoica Ciencia y Tecnología Agropecuaria 9 (2): 55-61.

KONDO, T.; RAMOS, A. A.; VERGARA, E. 2008b. Updated list of mealybugs and putoids from Colombia (Hemiptera: Pseudococcidae and Putoidae). Boletín del Museo de Entomología de la Universidad del Valle 9 (1): 29-53.

KOSZTARAB, M. 1996. Scale Insects of Northeastern North America: Identification, Biology and Distribution 3a ed. Virginia Museum of Natural History, Martinsville. 650 p.

KOSZTARAB, M.; KOZÁR, F. 1988. Scale insects of Central Europe 1a ed. Dr. W. Junk Publishers and Akadémia Kiado, Budapest, Hungría. $456 \mathrm{p}$.

KOZÁR, F. 2004. Ortheziidae of the World. Plant Protection Institute, Hungrian Academy of Science, Budapest. 525 p.

KOZÁR, F.; KONCZNÉ, Z. 2007. Rhizoecinae of the World 1a ed. Plant Protection Institute, Hungrian Academy of Science, Budapest. $617 \mathrm{p}$

LE PELLEY, R. H. 1973. Coffee insects. Annual Review of Entomology 18 (1): 121-142.

MALUMPHY, C. 2014. An annotated checklist of scale insects (Hemiptera: Coccoidea) of Saint Lucia, Lesser Antilles. Zootaxa 3846 (1): 69-86.

McKENZIE, H. L. 1967. Mealybugs of California with taxonomy, biology and control of North American species (Homoptera: Coccoidea: Pseudococcidae). University of California Press, Berkeley and Los Angeles. 525 p.

MILLER, D. R.; ARAPU, S. P. 1997. A new species of mealybug in the genus Dysmicoccus (Hemiptera: Coccoidea: Pseudococcidae) of importance in the eastern United States. Proccedings of the Entomological Society of Washington 99 (3): 440-460.

MILLER, G. L.; WILLIAMS, D. J. 2002. Dysmicoccus Ferris and similar genera (Hemiptera: Coccoidea: Pseudococcidae) of the Gulf State Region including a description of a new species and new United States records. Proceedings of the Entomological Society of Washington 104 (4): 968-979.
MOGHADDAM, M. 2013. A review of the mealybugs (Hemiptera: Coccoidea: Pseudococcidae, Putoidae and Rhizoecidae) of Iran, with descriptions of four new species and three new records for the Iranian fauna. Zootaxa 3632 (1): 1-107.

MURILLO, L. M. 1931. Los parásitos del café en el departamento de Antioquia. Revista Cafetera de Colombia 3: 943-949

ORELLANA, F.; VERA, H. 1989. Las cochinillas harinosas (Dysmicoccus brevipes Cockerell y Rhizoecus prob. americanus Hambleton) en viveros de palma africana y su control. Instituto Nacional de Investigaciones Agropecuarias, Quito. 7 p.

RAMOS, A. A.; SERNA, F. J. 2004. Coccoidea de Colombia, con énfasis en las cochinillas harinosas (Hemiptera: Pseudococcidae). Revista de la Facultad Nacional de Agronomía de Medellín 57 (2): 2383-2412.

RAMOS-PORTILlA, A. A.; CABALLERO, A. 2016. Rhizoecus colombiensis Ramos \& Caballero, a new species of hypogeal mealybug (Hemiptera: Coccomorpha: Rhizoecidae) and a key to the species of Rhizoecus from Colombia. Zootaxa 4092 (1): $55-68$.

RAMOS-PORTILlA, A. A.; CABALLERO, A.; KONDO, T.; SERNA. F. 2014. First record of Ripersiella kelloggi Ehrhon and Cockerell (Hemiptera: Rhizoecidae) for the Neotropics, with a redescription of the adult female. Insecta Mundi 876 (1): 1-7.

SANTA-CECILIA, L. V.; REIS, P. R.; SOUZA, C. 2002. Sobre a nomenclatura das espécies de cochonilhas-farinhentas do cafeeiro nos estados de Minas Gerais e Espírito Santo. Neotropical Entomology 31 (2): 333-334.

SCHNEIDER, S. A.; LAPOLLA, J. S. 2011. Systematics of the mealybug tribe Xenococcini (Hemiptera: Coccoidea: Pseudococcidae), with a discussion of trophobiotic associations with Acropyga Roger ants. Systematic Entomology 36 (1): 57-82.

SOUSA, A. L. V.; SOUZA, B.; SANTA-CECÍLIA, L. V. C.; PRADO, E. 2012. Especificidade alimentar: Em busca de um caráter taxonômico para a diferenciação de duas espécies crípticas de cochonilhas do gênero Planococcus (Hemiptera: Pseudococcidae). Revista Brasileira de Fruticultura 34 (3): 744-749.

TANAKA, H.; UESATO, T. 2012. New records of some potential pest mealybugs (Hemiptera: Coccoidea: Pseudococcidae) in Japan. Applied Entomology and Zoology 47 (4): 413-419.

VEA, I. M. 2014. Morphology of the males of seven species of Ortheziidae (Hemiptera: Coccoidea). American Museum Novitates 3812 (1): 1-36.

VILLEGAS, C.; BENAVIDES, P.; ZABALA, G.; RAMOS, A. A. 2009. Cochinillas harinosas asociadas a las raíces del café: descripción y biología. Avances Técnicos $\mathrm{N}^{\circ} 386$. Cenicafé, 8 p.

VILLEGAS-GARCİA, C.; BENAVIDES-MACHADO, P. 2011. Identificación de cochinillas harinosas en las raíces de café en departamentos cafeteros de Colombia. Revista Cenicafé 62 (1): 48-55.

WATSON, G. W.; COX, J. M. 1990. Identity of the African coffee root mealybug, with descriptions of two new species of Planococcus (Homoptera: Pseudococcidae). Bulletin of Entomological Research 80 (1): 99-105.

WATSON, G. W.; KUBIRIBA, J. 2005. Identification of mealybugs (Hemiptera: Pseudococcidae) on banana and plantain in Africa. African Entomology 13 (1): 35-47.

WILLIAMS, D. J. 1968. A revision of the genus Geococcus Green (Homoptera, Coccoidea, Pseudococcidae). Bulletin of Entomological Research 59 (1): 505-517.

WILLIAMS, D. J. 1985. Pseudococcus mandio sp. n. (Hemiptera: Pseudococcidae) on cassava roots in Paraguay, Bolivia and Brazil. Bulletin of Entomological Research 75 (1): 545-547.

WILLIAMS, D. J. 1998. Mealybugs of the genera Eumyrmococcus Silvestri and Xenococcus Silvestri associated with the ant genus Acropyga Roger and a review of the subfamily Rhizoecinae (Hemiptera, Coccidae, Pseudococcidae). Bulletin of the British Museum (Natural History) 67 (1): 1-152. 
WILLIAMS, D. J. 2004a. A synopsis of the subterranean mealybug genus Neochavesia Williams and Granara de Willink (Hemiptera: Pseudococcidae: Rhizoecinae). Journal of Natural History 38 (22): 2883-2899.

WILLIAMS, D. J. 2004b. Mealybugs of southern Asia. The Natural History Museum Kuala, Southdene SDN. BHD, Lumpu. 896 p.

WILLIAMS, D. J.; GRANARA DE WILLINK, M. C. 1992. Mealybugs of Central and South America 1a ed. CAB International, London, Reino Unido. 635 p.

WILLIAMS, D. J.; McKENZIE, H. L. 1971. Sixth taxonomic study of North America mealybugs, with additional species from South America (Homoptera: Coccoidea: Pseudococcidae). Hilgardia 40 (17): 565-602.

WILLIAMS, D. J.; GULLAN, P. J.; MILLER, D. R.; HAN, S. I. 2011. A study of the scale insect genera Puto Signoret (Hemip- tera: Sternorrhyncha: Coccoidea: Putoidae) and Ceroputo Šulc (Pseudococcidae) with a comparison to Phenacoccus Cockerell (Pseudococcidae). Zootaxa 2802 (1): 1-22.

Recibido: 3-feb-2017 • Aceptado: 2-jun-2017

Citación sugerida:

CABALlERO, A.; RAMOS-PORTILLA, A. A.; GIL, Z. N.; BENAVIDES, P. 2018. Insectos escama (Hemiptera: Coccomorpha) en raíces de café de Norte de Santander y Valle del Cauca, Colombia y descripción de una nueva especie. Revista Colombiana de Entomología 44 (1): 120-128. Enero - Junio 2018. 